\begin{tabular}{|c|c|}
\hline Title & A Single Spherical A ssembly of Protein A myloid Fibrils Formed by Laser Trapping \\
\hline Author(s) & Y uyama, Ken-ichi; U eda, Mariko; Nagao, Satoshi; Hirota, Shun; Sugiyama, Teruki; Masuhara, Hiroshi \\
\hline Citation & $\begin{array}{l}\text { A ngew andte chemie international edition, 56(24), 6739-6743 } \\
\text { https://doi.org/10.1002/anie.201702352 }\end{array}$ \\
\hline Issue Date & 2017-06-06 \\
\hline Doc URL & http:/hdl.handle.net/2115/70685 \\
\hline Rights & $\begin{array}{l}\text { This is the peer reviewed version of the following article: K. Y uyama, M. Ueda, S. Nagao, S. Hirota, T. Sugiyama, H. } \\
\text { Masuhara, A ngew. Chem. Int. Ed. 2017, 56, 6739, which has been published in final form at } \\
\text { http://dx.doi.org/10.1002/anie.201702352. This article may be used for non-commercial purposes in accordance with } \\
\text { Wiley Terms and Conditions for Self-A rchiving. }\end{array}$ \\
\hline Type & article (author version) \\
\hline Additional Information & There are other files related to this item in HUSCAP. Check the above URL. \\
\hline File Information & A Single Spherical A ssembly of Protein A myloid Fibrils Formed by Laser Trapping.pdf \\
\hline
\end{tabular}

Instructions for use 


\title{
A Single Spherical Assembly of Protein Amyloid Fibrils Formed by Laser Trapping
}

\author{
Ken-ichi Yuyama, ${ }^{[a]}{ }^{\#}$ Mariko Ueda, ${ }^{[b]}$ Satoshi Nagao, ${ }^{[b]}$ Shun Hirota, ${ }^{* b]}$ Teruki Sugiyama, ${ }^{*[a, b]}$ and \\ Hiroshi Masuhara*[a]
}

\begin{abstract}
Protein amyloids receive much attention due to their correlation with serious diseases and to their promising mechanical and optical properties as future materials. Amyloid formation has been conducted by tuning temperature and chemical conditions, so that its nucleation and the following growth are analyzed as ensemble dynamics. Here we succeeded in generating a single spherical assembly of amyloid fibrils of cytochrome $c$ domainswapped dimer upon laser trapping. The amyloid fibrillar structure was confirmed by fluorescence characterization and electron microscopic observation. The prepared spheres are further manipulated individually in solution to fabricate a three-dimensional micro-structure and a line pattern. Amyloid formation dynamics and amyloid-based micro-structure fabrication are demonstrated based on direct observation of a single spherical assembly, which foresees a new approach in amyloid studies.
\end{abstract}

Dynamics and mechanism of amyloid formation and crystallization of proteins have been studied comparatively, since both consist of nucleation and the following growth process where some time lag is usually observed. ${ }^{[1]}$ Amyloid nucleation is started from misfolded and unfold conformations of proteins and leads to fibrillation through mutual interactions of prefibrillar oligomer intermediates. ${ }^{[2]}$ Protein crystallization is coupled with the formation of protein clusters containing solvent waters and the subsequent generation of a highly concentrated area of such clusters. ${ }^{[3]}$ Nucleation and the crystal growth proceed in such an area of a few tens-a few hundreds of nanometers. Most of the experiments for amyloid formation and crystallization are carried out in solution by tuning $\mathrm{pH}^{[1]}$, salt concentration $^{[1]}$, and temperature ${ }^{[2]}$ and by applying ultrasonication $^{[3]}$, electromagnetic field ${ }^{[4]}$, and pulsed laser irradiation ${ }^{[5,6]}$. Therefore, all the processes of nucleation and growth of amyloid and crystal in solution proceed randomly in parallel, whose dynamic evolutions are monitored and analyzed as an ensemble of amyloid fibrils or crystals. It is considered very promising to propose a new experimental approach for preparing a single spherical assembly of protein amyloid, analyzing its dynamics, and fabricating micro-structures from

[a] Dr. K. Yuyama, Prof. T. Sugiyama, Prof. H. Masuhara Department of Applied Chemistry National Chiao Tung University

1001 University Road, Hsinchu 300, Taiwan

E-mail: sugiyama@g2.nctu.edu.tw, masuhara@masuhara.jp

[b] M. Ueda, Dr. S. Nagao, Prof. S. Hirota, Prof. T. Sugiyama

Graduate School of Materials Science

Nara Institute of Science and Technology

8916-5 Takayama-cho, Ikoma, Nara 630-0192, Japan

E-mail: hirota@ms.naist.jp

\# Present address: Research Institute for Electronic Science, Hokkaido University, N20W10, Kita-Ward, Sapporo 001-0020, Japan single assemblies. It will enable us to perform amyloid studies by watching always when and where individual assemblies of amyloid fibrils are prepared, monitored, and utilized.

Here we report a laser trapping study on oxidized monomeric horse cytochrome (cyt) $C$ and its domain-swapped dimer (Figs. $1 \mathrm{~A}$ and $1 \mathrm{~B})^{[7]}$. Cyt $c$ is a well-known globular heme protein that transfers electrons from cyt $b c 1$ complex to cyt $c$ oxidase in the respiratory chain of mitochondria. Monomeric cyt $c$ has three long $\alpha$-helices surrounding a protein-bound heme, ${ }^{[8,9]}$ whereas the domain-swapped dimeric cyt $c$ is formed by exchanging the $C$-terminal $\alpha$-helix with the corresponding unit of another cyt $c$ molecule. Laser trapping of cyt $c$ inside solution was already reported for monomeric bovine cyt $c$, where a particle-like assembly without structural change of constituent proteins was formed at the focal spot. ${ }^{[10]}$ On the other hand, it was reported that protein dimerization by domain-swapping tends to accelerate aggregation into amyloid-like fibrils. ${ }^{[11-13]}$ Examining these results, we considered that laser trapping-induced amyloid formation of the domain-swapped dimer is possible and designed a comparative study on monomer and dimer of horse heart cyt $c$.

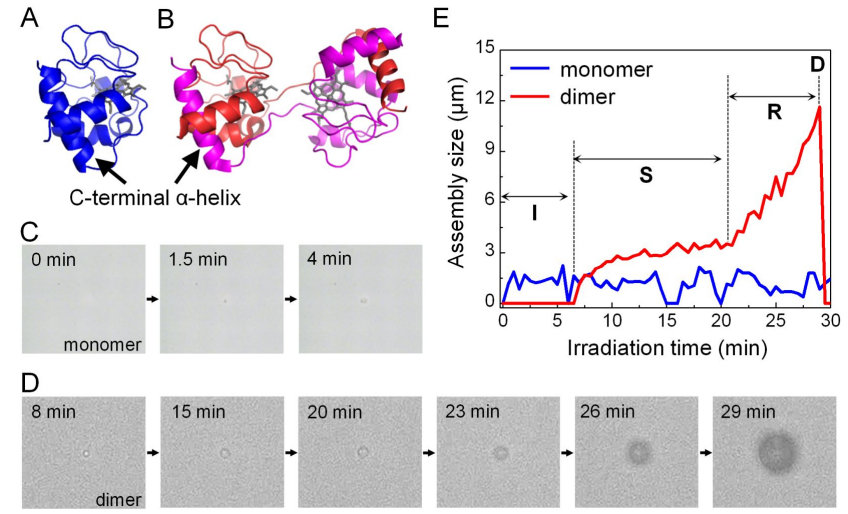

Figure 1. Laser trapping of cyt $c$. (A, B) Molecular structures of oxidized monomeric and domain-swapped horse cyt $c$. (C, D) Laser trapping of monomeric and dimeric cyt $c$. The size of images is $40 \times 35 \mu \mathrm{m}^{2}$. (E) The time evolution of the assembly size estimated from the captured images. I; incubation time, $\mathbf{S}$; saturation tendency, $\mathbf{R}$; rapid enlargement, $\mathbf{D}$; disappearance.

Laser trapping of a Rayleigh particle with the size smaller than the laser wavelength is described by Maxwell-Boltzmann theory and the force exerted on the particle is given as a function of electric field, magnetic flux density, and polarizability of the particle in solution. ${ }^{[14]}$ Under high irradiation, molecular diffusion should be slightly suppressed in optical potential, and the molecules can stay there longer, leading to formation of a transient complex by their mutual association. Due to a larger volume of the formed complex, optical potential becomes deeper, 
and by repeating this process protein molecules merge to a larger complex. ${ }^{[15]}$ In the present work, upon intense focused irradiation with $1064 \mathrm{~nm}$ laser in $\mathrm{D}_{2} \mathrm{O}$ solution, monomer cyt $c$ was confined in the focal volume and one small, particle-like assembly of $1.5 \mu \mathrm{m}$ in diameter was formed (Figs. 1C and $1 \mathrm{E}$ ). This phenomenon is similar to laser trapping assembling of polymer chains, amino acids, and a few native proteins. ${ }^{[10,15-18]}$ As cyt $c$ is positively charged, their mutual electrostatic repulsion should be overcome by optical potential, which was already proven for synthetic polyelectrolyte. ${ }^{[18]}$

The dimeric cyt $c$ was initially not trapped, but a single assembly with the size of $1.5 \mu \mathrm{m}$ was observed at a few min after starting the trapping (Fig. 1D). We examined this trapping and the following growth for 5 samples and confirmed its stochastic characteristics, which is summarized in Fig. 1E (see Supporting Information for other samples). The incubation time before the single assembly became visible (I), the saturation tendency in the size increase (S), the rapid growth to $10 \mu \mathrm{m}(\mathbf{R})$ are changed from sample to sample. On the other hand, the sizes when the assembly appears and disappears were always $1.5 \mu \mathrm{m}$ and about $10 \mu \mathrm{m}$, respectively.

To characterize the single assembly formed from the dimeric cyt $c$, the sphere just before disappearance (D in Fig. 1E) was analyzed. It was manipulated to a glass substrate with hydrophobic surface by lowering the focal position manually. The assembly was adsorbed tightly to the glass substrate and remained stably there even after stopping the laser trapping. It could not be detached again by optical manipulation with the same laser, indicating that the assembly surface is hydrophobic.
After the spherical assembly was deposited on the glass substrate, a $D_{2} \mathrm{O}$ solution of thioflavin $\mathrm{T}$ (ThT) was added to the solution and the obtained solution was exposed to 405-nm laser light. The fluorescence intensity of the assembly gradually increased during the observation (Figs. $2 \mathrm{~A}$ and 2B). The obtained fluorescence spectrum with a peak around $490 \mathrm{~nm}$ is similar to that of ThT solution containing protein amyloids, ${ }^{[19]}$ indicating that the sphere consists of amyloid fibrils.

We conducted electron microscopy of the dimer assembly to directly confirm its amyloid fibrillar structures. For scanning electron microscopy (SEM), we deposited the assembly of dimeric cyt $c$ and extended it linearly by shifting the focal point (Fig. 2C) repetitively. The results obtained with different resolution are summarized in Fig. 2D, where no spherical shape was observed anymore. The fibrillar structures of several tens of nanometers in width and longer than several hundred nanometers in length were observed, and appeared to be tangled with each other, forming their lump. For transmission electron microscopy (TEM), we washed the linearly extended dimer lump (Figure 2D) with $\mathrm{D}_{2} \mathrm{O}$, sonicated it, and prepared its fragments. The observed results and a corresponding illustration are shown in Fig. $2 \mathrm{E}$ and $2 \mathrm{~F}$, where the width of the constitutive fibrils was $5-10 \mathrm{~nm}$ and bundles consisting of loosely tangled fibrils were observed. The width of the bundles was 100-150 nm and the length was longer than several hundred nanometers. The characteristics of this TEM observation corresponded well to the SEM images and match with the structures of amyloid fibrils.

Now amyloid formation dynamics is considered based on the
A
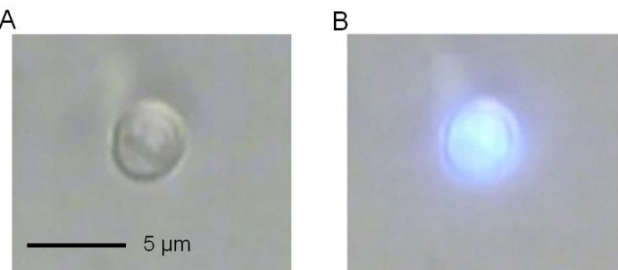

D
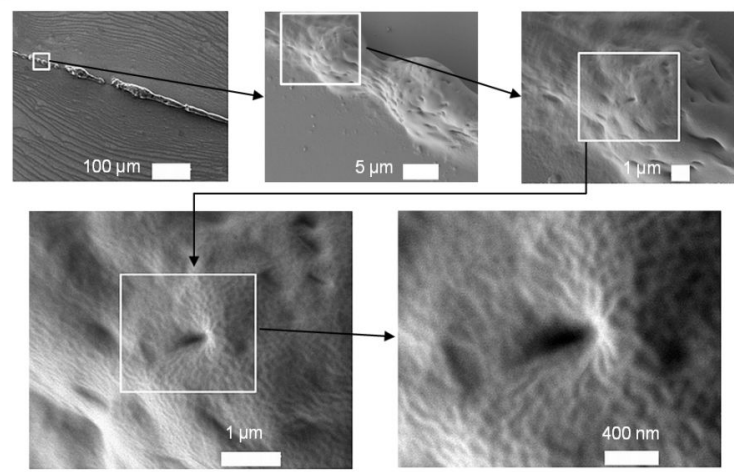

C

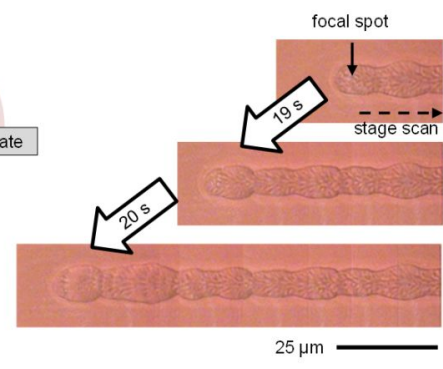

E
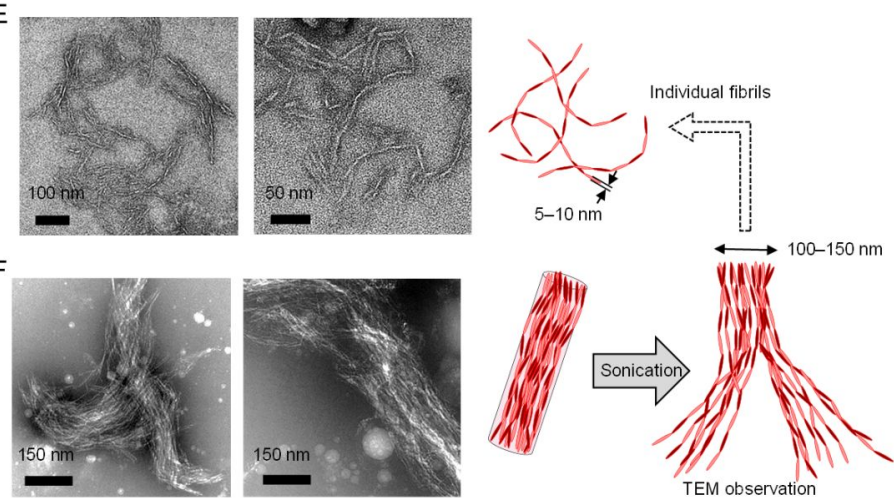

Figure 2. Characterization of prepared amyloid. (A) A spherical assembly of dimeric cyt $c$ in $\mathrm{D}_{2} \mathrm{O}$ solution formed by laser trapping. (B) The assembly stained with ThT and exposed to the UV laser. (C) A procedure for preparing a linearly extended structure by laser trapping of dimeric cyt $c$. (D) SEM images of its linear assembly. TEM images and a schematic illustration of $(E)$ amyloid fibrils and (F) their bundles of dimeric cyt $c$. 
direct observation of a single growing assembly of the dimer at the focus. Assembling rate of nanoparticles is generally determined by their size, concentration, and diffusion. As the dimer solution was adjusted to have a concentration of heme chromophore same to that of monomer cyt $c$, the concentration of the dimer was half compared to the monomer. The formation rate of the dimer assembly should be decreased to a half compared to the monomer cyt $c$, however, the sphere-like assembly was always observed only after I. We consider that, as the dimer is more positively charged compared to the monomer, the dimer is initially very repulsive with each other and shows no gathering behavior. Under strong confinement due to the laser trapping, the domain-swapped dimer may undergo some conformational and/or structural change, suppressing the repulsive interaction between the dimers. Then their mutual association proceeds rapidly to generate an assembly with the size of $1.5 \mu \mathrm{m}$ in diameter. This is considered to determine the characteristic value of $\mathbf{I}$.

Next, dynamic behavior of $\mathbf{S}$ and $\mathbf{R}$ is elucidated referring our systematic study of laser trapping crystallization of amino acid. Laser trapping collects a number of molecular clusters of amino acid and their efficient association gives a single highly concentrated large area of amino acid clusters. ${ }^{[15]}$ This idea was confirmed by directly observing crystallization behavior of Lphenylalanine under laser trapping conditions. ${ }^{[20]}$ Here we infer that domain-swapped dimer cyt $c$ similarly forms a highly concentrated protein area, which proceeds during $\mathbf{S}$ accompanying a small increase in the assembly size. In this formed area the growth of the single assembly is accelerated giving a diameter of about $10 \mu \mathrm{m}(\mathbf{R})$.

It is an important and interesting issue when the amyloid was nucleated. One interpretation is that the nucleation starts during I and evolves to a single assembly consisting of amyloid fibrillar structures. Its surface reacts efficiently with the dimer clusters in the concentrated area through $\mathbf{S}$ and $\mathbf{R}$, expanding as an assembly of the amyloid fibrils. Another interpretation is that amyloid nucleation takes place during $\mathbf{S}$ and/or $\mathbf{R}$. A sphere of $1.5 \mu \mathrm{m}$ absorbs the clusters from the highly concentrated area and grows forming a more packed structure. This packing may be suitable for nucleation and such fibril formation at a certain position should propagate over the packed assembly, giving a large sphere of amyloid fibrils just before $\mathbf{D}$.

A

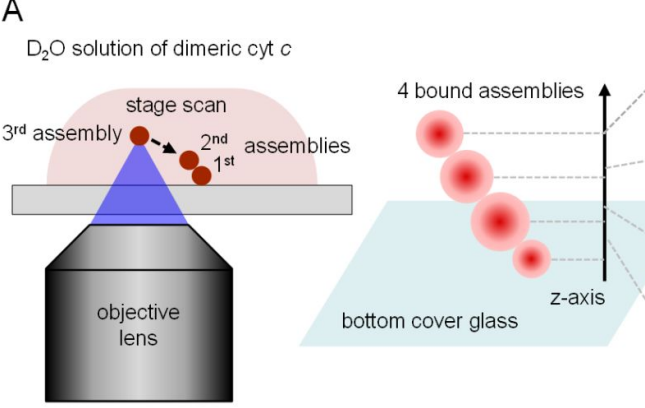

At present, we cannot determine which interpretation is responsible for the amyloid formation, while a possible molecular picture of amyloid formation can be considered in terms of polymerization. It is reported that the cyt $c$ dimer is polymerized by successive domain swapping, ${ }^{[7]}$ on which we assume such a reaction characteristic of the domain-swapped dimer is related to the nucleation. Association of the dimers to the nucleus and the sequential reaction correspond to amyloid growth in the highly concentrated area. Larger size of an assembly of the fibrils receive stronger optical force due to larger volume accelerating the growth process, which could be auto-catalytic and examined by in-situ microspectroscopic analysis during the trapping.

The present laser trapping experiment has high potential in fabricating microstructures of protein amyloid fibrils in solution. First, we prepared one assembly and deposited it on the glass surface by optical manipulation, and then another was similarly prepared and manipulated toward a position obliquely above the initial assembly. By repeating this procedure, four spherical assemblies were connected to each other and interestingly held at an angle of approximately 60 degrees relative to the glass substrate (Fig. $3 \mathrm{~A}$ ). The rigid standing structure means that the assembly possessed not only a highly hydrophobic surface but also a strong inner rigid structure. Such 3D construction of microstructures in solution has never been reported for amyloidbased materials as far as we know.

Second, we prepared a single line pattern of amyloid fibrils with the length of $1 \mathrm{~mm}$ and the width of a few tens micrometers by repeating the procedure shown in Fig. $2 \mathrm{C}$ and evaluated it under the crossed polarization conditions (Fig. 3B). No specific image of the line could be observed when the long axis of the assembly was identical to the direction of a polarizer or an analyzer. Upon rotating the sample, a clear line pattern appeared, indicating that amyloid fibrils were aligned to a certain degree in the line. This anisotropy should be ascribed to the preparation process where a single sphere of amyloid assembly was pressed to the surface by shifting the microscope stage. The spherical shape was much deformed and pulled along the shifting direction, where fibrillar structures were oriented. This is similar to flow-induced alignment of amyloid protofilaments, ${ }^{[21]}$ and reminds us that mechanically extended polymer films

Figure 3. Characteristics of amyloid assembly. (A) A procedure for preparing a three-dimensional structure of dimeric cyt $C$ assemblies by laser trapping and optical micrographs of four connected assemblies along an oblique direction. (B) Transmission images of the linearly extended assemblies with and without crossed polarizers. The directions of a polarizer and an analyzer are indicated in the image. The line was set at 0,45 , and 90 degrees against the direction of the analyzer. The size of images is $400 \times 300$ $\mu \mathrm{m}^{2}$. 
sometimes show birefringence and widely used. Here the spatial resolution of amyloid patterning is low, but it will be improved as we have demonstrated for laser trapping of single gold nanoparticles. ${ }^{[22]}$

Generally, amyloids are prepared in solution, and then collected and used for constructing microstructures. ${ }^{[23,24]}$ The present experiment enables us to prepare the single spherical assembly consisting of protein amyloid fibrils and spatially arrange them in the same solution by using the same laser. The detailed analysis and characterization of the spheres and microstructures should be carried out individually and in-situ in solution by X-ray diffraction, NMR, CD, and FT-IR measurements, however, they are difficult under a microscope, while Raman microspectroscopy is promising which is being started in our laboratory. Here we have come to the stage that formation dynamics of protein amyloid fibrils can be examined by direct observation of the single sphere.

It is reported that fibrillar nanostructures can be produced not only for proteins and peptides but also for polymers. Due to the structure, amyloids are expected to have steel-level mechanical strength $^{[25]}$ in addition to various optical properties such as multiphoton absorption ${ }^{[26]}$ and optical anisotropy ${ }^{[27]}$. Until now such amyloid-based materials are prepared by the sequential processes of 1) amyloid formation by ultrasonication, heating, and $\mathrm{pH}$ change, and 2) their construction of films and patterns through casting, spin-coating, deposition to patterned substrate, and so on. Recently a new growth and shrinking control method of molecular assemblies was demonstrated for preparing peptide nanotubes by utilizing microfluidics and micropillars, ${ }^{[28]}$ which may be applied to amyloid formation. Our approach of laser trapping has an advantage of spatio-controllability in understanding dynamic aspects of materials preparation from amyloid fibrils and in analyzing amyloid-based material as functions of their size and shape. Furthermore polymorph control of amyloid by laser trapping is expected, which is supported by our work on laser trapping-controlled crystal polymorph of amino acids. ${ }^{[15]}$ Laser trapping will play important roles in studying fundamental dynamics and mechanism of amyloid formation and in designing novel bottom-up nanomaterials from amyloids.

\section{Acknowledgements}

The present work is partly supported by the Ministry of Education, Taiwan ("Aiming the Top University Plan" of National Chiao Tung University) and the Ministry of Science and Technology, Taiwan (Grants to T.S. (MOST 105-2113-M-492001-), and to H.M. (MOST 105-2811-M-009-022)), and JSPS KAKENHI Grant Number JP16H06507 in Scientific Research on Innovative Areas "Nano-Material Optical-Manipulation" to T.S., and Japan Society for the Promotion of Science (S.H. (CategoryB, No. JP26288080; Innovative Areas, JP16H00839).
Keywords: laser trapping $\bullet$ amyloid $\bullet$ nucleation $\bullet$ spatiotemporal formation $\cdot$ domain-swapped protein

[1] D. Maes, M. A. Vorontsova, M. A. C. Potenza, T. Sanvito, M. Sleutel, M Giglio, P. G. Vekilov, Acta Cryst. 2015, F71, 815-822.

[2] E. Chatani, H. Imamura, N. Yamamoto, M. Kato, J. Biol. Chem. 2014 289, 10399-10410.

[3] Yoshimura, M. So, K. Sakurai, H. Yagi, Y. Goto, Biochim. Biophys. Acta 2013, 1834, 2640-2646.

[4] G. Sazaki, E. Yoshida, H. Komatsu, T. Nakada, S. Miyashita, K. Watanabe, J. Cryst. Growth 1997, 173, 231-234.

[5] B. A. Garetz, J. E. Aber, N. L. Goddard, R. G. Young, A. S. Myerson, Phys. Rev. Lett. 1996, 77, 3475-3476.

[6] H. Adachi, K. Takano, Y. Hosokawa, T. Inoue, Y. Mori, H. Matsumura, M. Yoshimura, Y. Tsunaka, M. Morikawa, S. Kanaya, H. Masuhara, Y. Kai, T. Sasaki, Jpn. J. Appl. Phys. 2003, 42, L798-L800.

[7] S. Hirota, Y. Hattori, S. Nagao, M. Taketa, H. Komori, H. Kamikubo, Z Wang, I. Takahashi, S. Negi, Y. Sugiura, M. Kataoka, Y. Higuchi, Proc Natl. Acad. Sci. USA 2010, 107, 12854-12859.

[8] R. E. Dickerson, T. Takano, D. Eisenberg, O. B. Kallai, L. Samson, A Cooper, E. Margoliash, J. Biol. Chem. 1971, 246, 1511-1535.

[9] G. W. Bushnell, G. V. Louie, G. D. Brayer, J. Mol. Biol. 1990, 214, 585595.

[10] Y. Tsuboi, T. Shoji, M. Nishino, S. Masuda, K. Ishimori, N. Kitamura Appl. Surf. Sci. 2009, 255, 9906-9908.

[11] J. M. Louis, I.-J. L. Byeon, U. Baxa, A. M. Gronenborn, J. Mol. Biol. 2005, 348, 687-698.

[12] M. Wahlbom, X. Wang, V. Lindström, E. Carlemalm, M. Jaskolski, A. Grubb, J. Biol. Chem. 2007, 282, 18318-18326.

[13] E. Zerovnik, V. Stoka, A. Mirtic, G. Guncar, J. Grdadolnik, R. A Staniforth, D. Turk, V. Turk, FEBS J. 2011, 278, 2263-2282.

[14] Y. Harada, T. Asakura, Opt. Commun. 1996, 124, 529-541.

[15] T. Sugiyama, K. Yuyama, H. Masuhara, Acc. Chem. Res. 2012, 45, 1946-1954.

[16] P. Borowicz, J. Hotta, K. Sasaki, H. Masuhara, J. Phys. Chem. B 1998, 102, 1896-1901.

[17] W. Singer, T. A. Nieminen, N. R. Heckenberg, H. Rubinsztein-Dunlop, Phys. Rev. E, 2007, 75, 011916-1-011916-5.

[18] J. Hotta, K. Sasaki, H. Masuhara, Y. Morishima, J. Phys. Chem. B 1998 102, 7687-7690.

[19] M. Biancalana, S. Koide, Biochim. Biophys. Acta 2010, 1804, 14051412

[20] K. Yuyama, J. George, K. G. Thomas, T. Sugiyama, H. Masuhara, Cryst. Growth Des. 2016, 16, 953-960.

[21] R. Adachi, K. Yamaguchi, H. Yagi, K. Sakurai, H. Naiki, Y. Goto, J. Biol Chem. 2007, 282, 8978-8983.

[22] S. Ito, H. Yoshikawa, H. Masuhara, Appl. Phys. Lett. 2002, 80, 482-484

[23] P. Mesquida, D. L. Ammann, C. E. MacPhee, R. A. Mckendry, Adv. Mater. 2005, 17, 893-897.

[24] V. Dinca, E. Kasotakis, J. Catherine, A. Mourka, A. Ranella, A Ovsianikov, B. N. Chichkov, M. Farsari, A. Mitraki, C. Fotakis, Nano Lett. 2008, 8, 538-543.

[25] T. P. Knowles, A. W. Fitzpatrick, S. Meehan, H. R. Mott, M. Vendruscolo, C. M. Dobson, M. E. Welland, Science 2007, 318, 19001903.

[26] P. Hanczyc, M. Samoc, B. Norden, Nat. Photon. 2013, 7, 969-972.

[27] T. P. J. Knowles, T. W. Oppenheim, A. K. Buell, D. Y. Chirgadze, M. E. Welland, Nat. Nanotechnol. 2010, 5, 204-207.

[28] Z. A. Arnon, A. Vitalis, A. Levin, T. C. T. Michaels, A. Caflisch, T. P. J. Knowles, L. Adler-Abramovich, E. Gazit, Nat. Commun. 2016, 7, 13190. 
Entry for the Table of Contents (Please choose one layout)

\section{COMMUNICATION}

Single amyloid assembly formation by light: Protein amyloids receive much attention due to their correlation with serious diseases and to their promising mechanical and optical properties as future materials. A single spherical assembly of amyloid fibrils is prepared in a spatio-temporally controlled manner by laser trapping. Amyloid formation dynamics and amyloid-based microstructure fabrication are demonstrated, which foresees a new approach in amyloid studies.

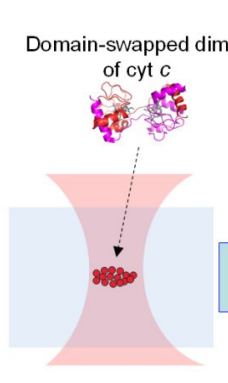

Laser trapping and assembling
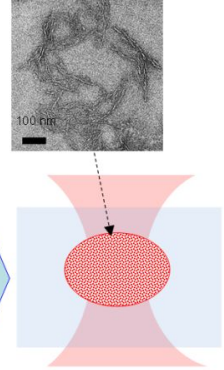

Single spherical assembly of amyloid fibrils
Author(s), Corresponding Author(s)*

Page No. - Page No.

Title 